\title{
Abnormal fermentations in continuous cultures of rumen micro-organisms given cobalt-deficient hay or barley as the food substrate
}

\author{
By P. MCDONALD AND N. F. SUTTLE \\ Moredun Research Institute, 408 Gilmerton Road, Edinburgh EH17 $7 J H$
}

(Received 2 October 1985 - Accepted 3 April 1986)

\begin{abstract}
1. Four experiments, each with four concurrent continuous cultures of rumen micro-organisms, were used to investigate the effects of inoculum, food substrate and cobalt supplementation on the course of fermentation and vitamin $B_{12}$ synthesis. The inocula came from sheep receiving either a Co-rich, complete diet (Ruminant $A$ : Expts 1, 2 and 4) or a Co-deficient hay (Expt 3). In Expt 2, inocula from different donors were used for each culture but for other experiments they were pooled. Co-deficient hay was used as the initial substrate and Co-supplements were given after 8-54 d Co-depletion.

2. In three of four experiments, two using a pooled inoculum, uncharacteristically low acetate (Ac) and high propionate $(\mathrm{Pr})$ outputs were obtained from the hay substrate in some cultures. In all, six cultures gave Ac: $\mathrm{Pr}$ values in the effluent $<2: 1$ and, in Expt 3, the differences remained evident for at least $6 \mathrm{~d}$ after a change to a Co-deficient barley substrate.

3. Abnormal cultures gave lower cobalamin (cbl) and vitamin $B_{12}$ analogue outputs than normal cultures: when supplemented with Co they showed small responses in cbl and large responses in analogue output with a slow increase in Ac: $\mathrm{Pr}$.

4. We suggest that the use of Co-deficient substrates led to shifts in the microbial populations of some cultures, indicating the $\mathrm{cbl}$-dependence of some species of rumen micro-organisms.
\end{abstract}

Rumen bacteria have a considerable capacity for incorporating dietary cobalt into corrinoid compounds but predominantly these lack the 5,6-dimethylbenzimidazole base which is essential for coenzyme activity in the host (e.g. Dryden et al. 1962; Dryden \& Hartman, 1971). Considerable attention has, therefore, been given to factors which influence the partition of Co between the coenzyme and analogous forms of vitamin $B_{12}$, and their possible implications for the host's nutrition (e.g. Gawthorne, 1970; Sutton \& Elliot, 1972). The two coenzymes which are active in mammalian tissues, methyl and adenosyl cobalamin (cbl) can, however, perform similar functions in bacteria: methyl cbl is a vital methyl donor in anaerobic methanogenic bacteria (Poston \& Stadtman, 1975) and adenosyl cbl is needed to support methylmalonyl-CoA mutase $(E C 5.4 .99 .2)$ activity and hence propionate metabolism in Propioni bacteria (Babior, 1975). It is, therefore, conceivable that some species of rumen bacteria are cbl-dependent and that partition of Co between coenzyme and analogous forms of vitamin $B_{12}$ influences microbial as well as host nutrition. We attempted to use a simple system for continuously culturing rumen micro-organisms (RUSITEC, Czerkawski \& Breckenridge, 1977) to gain more information about the factors which might influence vitamin $B_{12}$ synthesis in the rumen. We used Co-deficient foods as substrates with the intention of subsequently studying the partition of Co supplements between cbl and analogue synthesis but had difficulties in producing stable fermentations and sustaining acetate production.

MATERIALS AND METHODS

Culture system

In each of four experiments, the four concomitant cultures of RUSITEC were initiated and maintained in the manner described by Czerkawski \& Breckenridge (1977), using one of 
Table 1. Summary of inocula, substrates, cobalt depletion periods and Co treatments used in four experiments each with four concurrent RUSITEC cultures

\begin{tabular}{|c|c|c|c|c|c|c|}
\hline $\begin{array}{l}\text { Expt } \\
\text { no. }\end{array}$ & Inoculum & Substrate & 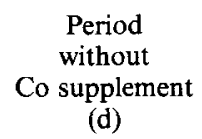 & $\begin{array}{c}\text { Vessel } \\
\text { no. }\end{array}$ & \multicolumn{2}{|c|}{$\begin{array}{l}\text { Co treatment } \\
(\mathrm{nmol} / \mathrm{d})\end{array}$} \\
\hline 1 & $\begin{array}{l}\text { One donor } \\
\text { on Ruminant } \\
\text { A }\end{array}$ & Hay 1 & 54 & $\begin{array}{l}1 \\
2 \\
3 \\
4\end{array}$ & $\left.\begin{array}{l}14 \cdot 3 \\
28 \cdot 5 \\
28 \cdot 5 \\
57 \cdot 1\end{array}\right\}$ for & $22 \mathrm{~d}$ \\
\hline 2 & $\begin{array}{l}\text { Four donors } \\
\text { on Ruminant } \\
\text { A }\end{array}$ & Hay 1 & 11 & $\begin{array}{l}1 \text { and } 2 \\
3 \text { and } 4\end{array}$ & $\left.\begin{array}{l}47 \cdot 5 \\
23 \cdot 8\end{array}\right\}$ for & $13 \mathrm{~d}$ \\
\hline 3 & $\begin{array}{l}\text { Pooled from } \\
\text { four donors } \\
\text { on hay } 1\end{array}$ & $\begin{array}{l}\text { Hay } 1 \\
\text { Barley }\end{array}$ & $\begin{array}{r}6 \\
17\end{array}$ & $\begin{array}{c}1-4 \\
1-2 \\
3 \text { and } 4\end{array}$ & $\begin{array}{r}\text { None } \\
11.9 \text { then } 47.6 \\
23.8 \text { then } 95.1\end{array}$ & for $14 \mathrm{~d}$ \\
\hline 4 & $\begin{array}{l}\text { Pooled } \\
\text { from four } \\
\text { donors on } \\
\text { Ruminant A }\end{array}$ & Hay 2 & 8 & $\begin{array}{l}1 \\
2 \\
3 \\
4\end{array}$ & $\left.\begin{array}{c}255 \text { as chalk } \\
8 \text { as sand } \\
770 \text { as clay } \\
20 \text { as loam }\end{array}\right\}$ & $\begin{array}{l}\text { given at } \\
0.7 \mathrm{~g} / \mathrm{d} \\
\text { for } 14 \mathrm{~d}\end{array}$ \\
\hline
\end{tabular}

Ruminant A, Co-rich diet (65 $\mu \mathrm{mol} \mathrm{Co/kg} \mathrm{DM;} \mathrm{Wainman} \mathrm{et} \mathrm{al.} \mathrm{1970).}$

two Co-deficient hays, containing 0.54 (hay 1) and 0.83 (hay 2) $\mu \mathrm{mol} \mathrm{Co} / \mathrm{kg}$ dry matter (DM), or a Co-deficient barley, containing $0 \cdot 19 \mu \mathrm{mol} \mathrm{Co} / \mathrm{kg} \mathrm{DM}$, at $7 \mathrm{~g} / \mathrm{d}$ as substrates. The dilution rate for artificial saliva (McDougall, 1948) was $0.75 / \mathrm{d}$ and $48 \mathrm{~h}$ were allowed for the digestion of each day's feed.

\section{Design of experiments}

Each experiment consisted of a preliminary Co-depletion period followed by periods of Co-supplementation (Analar cobalt nitrate; BDH Ltd, Poole, Dorset); the details are summarized in Table 1.

Expt 1 was intended to investigate changes in vitamin $B_{12}$ synthesis and fermentation in vitro following the supplementation of a Co-deficient hay with $\mathrm{Co}$. However, one culture had to be re-established after $11 \mathrm{~d}$ when the vessel began to leak: it was re-inoculated with rumen contents from a different donor on the same Co-rich diet (Ruminant A; $65 \mu \mathrm{mol}$ $\mathrm{Co} / \mathrm{kg} \mathrm{DM}$; Wainman et al. 1970). The characteristics of the new culture were so distinctive that it was decided to see how long this individuality was sustained.

Expt 2 investigated the influence of the individual donor on the culture established: each culture was inoculated with rumen contents from a different donor given Ruminant A. No differences in fermentation were apparent after $11 \mathrm{~d}$ and the responses to Co supplements were therefore studied.

In Expt 3, donors were given the low-Co hay (hay 1) for several weeks instead of Ruminant $\mathrm{A}$ in an attempt to hasten the Co depletion of the cultures and the same low-Co hay was also used initially as the culture substrate. After $6 \mathrm{~d}$, all cultures were given unsupplemented Co-deficient barley for $18 \mathrm{~d}$ before supplementation with $\mathrm{Co}$.

Expt 4 investigated variations in fermentation with the second Co-deficient hay as substrate but using pooled rumen contents from sheep on Ruminant $A$ to inoculate the cultures. Results for the Co-repletion period when different soils were used to provide Co are given elsewhere (McDonald \& Suttle, 1983). 


\section{Sampling of cultures}

Samples of liquid were taken every 1-3 d either from the culture vessel before feeding (Expts 1 and 2) or from the effluent which had collected over $24 \mathrm{~h}$, i.e. the total output (Expts 3 and 4): the collection flask contained $5 \mathrm{ml} 0.06 \mathrm{M}$-sodium cyanide to inhibit microbial metabolism and convert cbl to the stable cyano-forms.

\section{Analytical methods}

The competitive protein binding ( $\mathrm{cpb}$ ) radioassay used for determining cbl values was modified from Green (1980), purified Intrinsic Factor (Sigma, Poole, Dorset) replacing chicken serum as the binding agent. Total vitamin $B_{12}$ was estimated by the microbial method detailed by DIFCO Laboratories, Surrey, using Lactobacillus leichmanii ATCC 7830 (Torry Research Station, Aberdeen). Cbl concentrations were deducted from the total vitamin $B_{12}$ concentrations to give the concentration of vitamin $B_{12}$ analogues in each sample.

Volatile fatty acid (VFA) analysis was by gas-liquid chromatography using a GCD chromatograph (Pye Unicam, Cambridge). For samples from Expt 1, the separations were made with a $1.83 \mathrm{~m} \times 2 \mathrm{~mm}$ i.d. glass column containing SP-1200 phosphoric acid $(10: 1 \mathrm{w} / \mathrm{w})$ on $80 / 100$ Chromosorb WAW (Supelco; Houston, Texas) packing. For the other experiments, separation was by FFAP (100 g/l) on 100/120 Diatomite CQ (Supelco) packing in a $1.5 \mathrm{~m} \times 4 \mathrm{~mm}$ i.d. glass column; both columns allowed estimation of VFA up to C-5, including isomers. All unknowns and standards were treated (4:1) with $\mathrm{H}_{3} \mathrm{PO}_{4}$ and centrifuged ( $2000 \mathrm{~g}$ for $15 \mathrm{~min}$ ) before the supernatant was analysed. Cobalt analyses of both soil and food material were performed using flameless atomic absorption spectrophotometry. The apparent DM digestibility (ADMD) of the food substrate was estimated by drying the digested food to constant weight after incubation for $48 \mathrm{~h}$.

\section{RESULTS}

The principal attributes of the cultures in each of the experiments are summarized in Table 2. For clarity the results for different levels of Co supplementation within cultures have been pooled: none of the differences was large or statistically significant.

\section{Expt 1}

The distinctive nature of the fermentation established in culture 2, renewed from a different donor after $11 \mathrm{~d}$, is shown in Table 2. The culture quickly adopted a lower ADMD, acetate (Ac) and cbl concentration but higher propionate $(\mathrm{Pr})$, butyrate and valerate concentrations than were found previously in that vessel, and in all these characteristics it remained distinct from the other cultures for $40 \mathrm{~d}$ : Ac: $\operatorname{Pr}(2: 1)$ was below that typical of a roughage fermentation $(>3 \cdot 0)$. Supplementation of the culture with Co did not increase cbl concentrations or restore a completely normal fermentation although there was a rise in Ac: $\operatorname{Pr}($ Table 2).

\section{Expt 2}

The uniform initial fermentation characteristics of the four cultures are shown in Fig. 1 and Table 2. There was no evidence that the use of inocula from different donors markedly influenced the fermentation characteristics of the cultures despite differences in their initial cbl concentration. Total VFA concentrations in the culture fluid decreased rapidly over the first $5 \mathrm{~d}$, despite increases in the ADMD. Cbl concentrations also decreased rapidly between days 3 and 5 but slowly thereafter so that cbl:VFA in the culture fluid was still declining after $11 \mathrm{~d}$, reflecting the continuing Co-depletion of the cultures. Supplementation with Co 


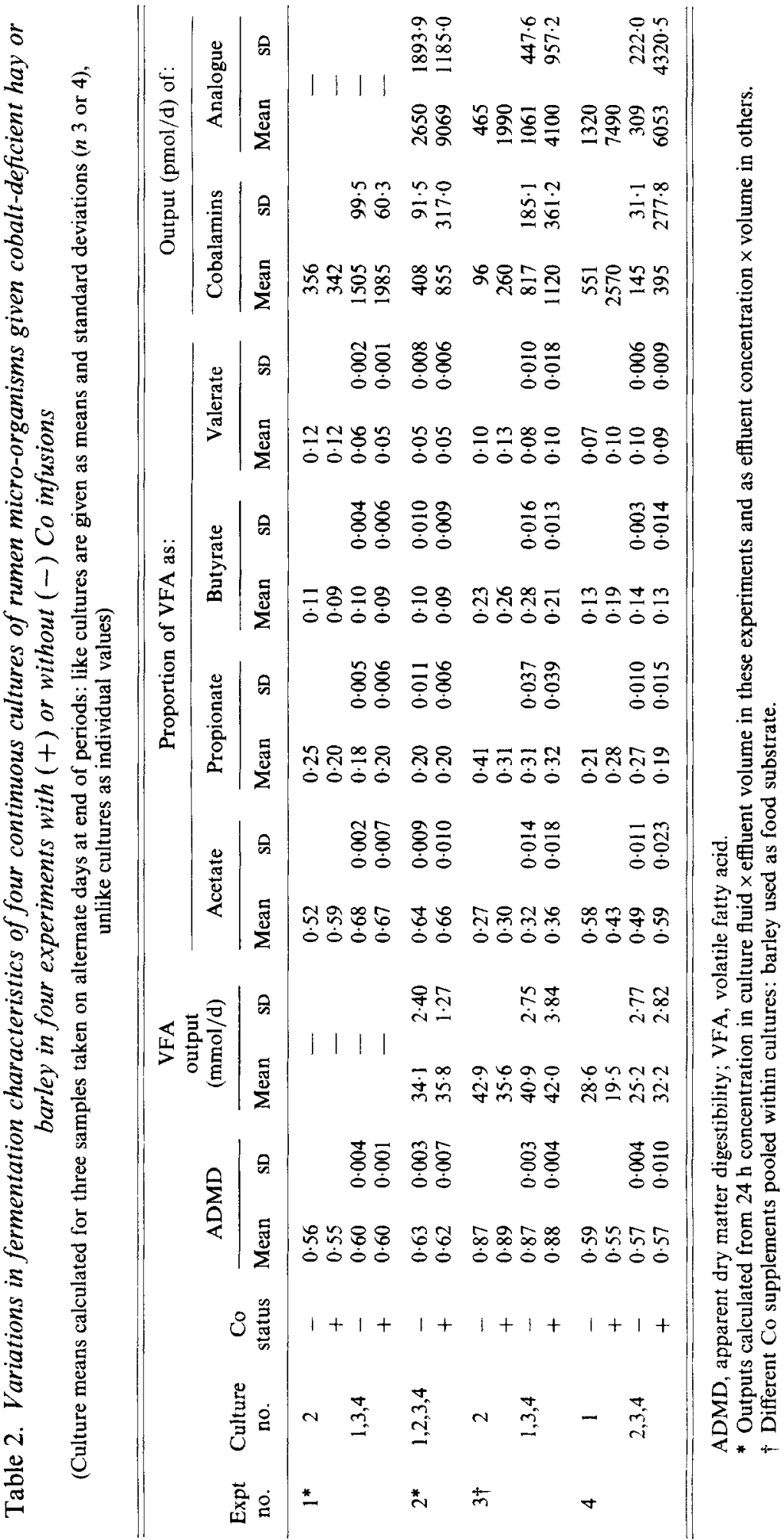



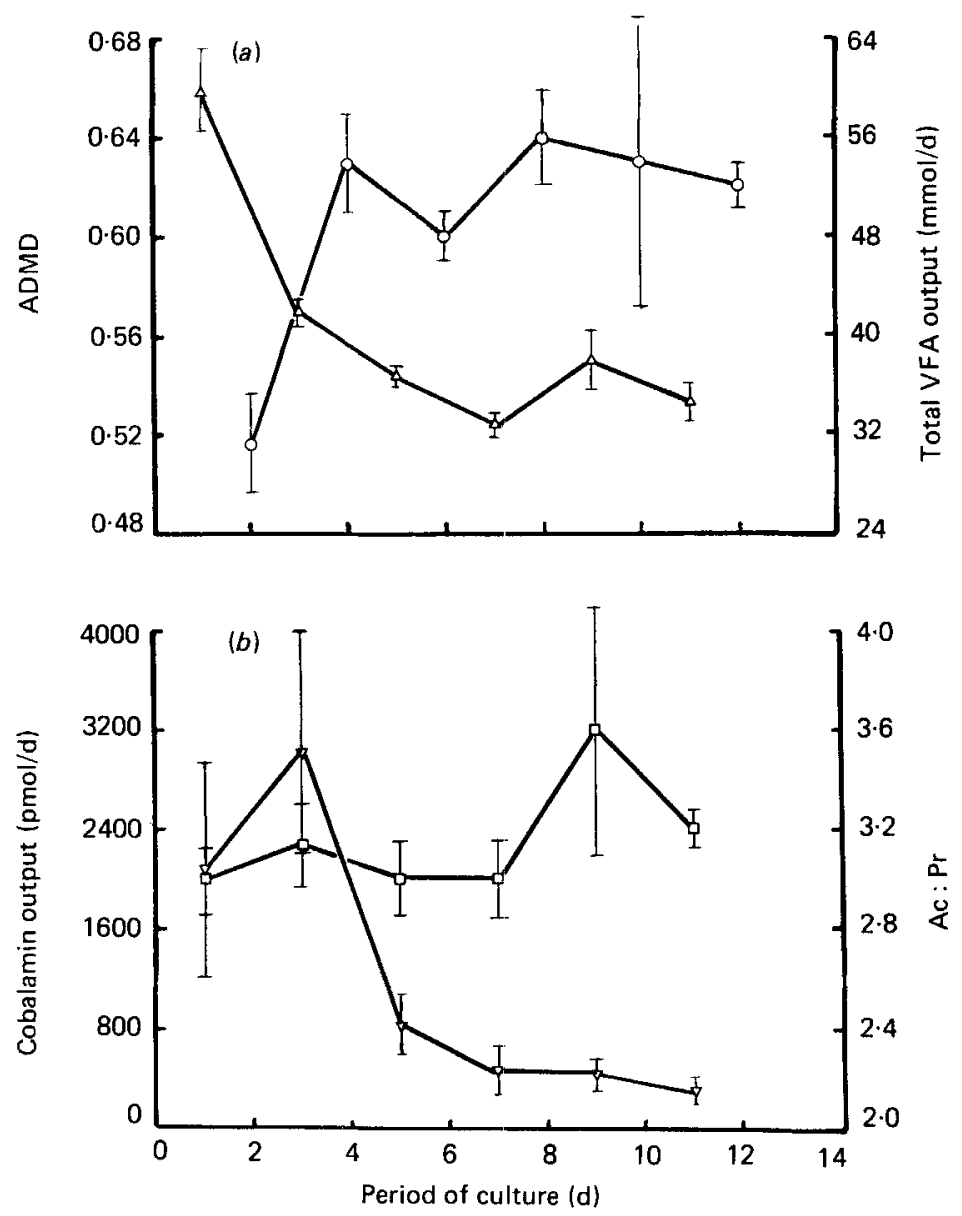

Fig. 1. Expt 2. The fermentation characteristics for the four RUSITEC cultures given cobalt-deficient hay as the food substrate; $(a)$ apparent dry matter digestibility (ADMD) (O) and total volatile fatty acid (VFA) output after $24 \mathrm{~h}(\triangle),(b)$ cobalamin output $(\nabla)$ and acetate: propionate $(A c: \operatorname{Pr}, \square)$. Points are mean values, and standard deviations are represented by vertical bars.

increased cbl and analogue outputs by similar proportions without affecting the products of fermentation (Table 2), Ac:Pr being characteristic of a roughage fermentation in all cultures $(3 \cdot 3$, SD $0 \cdot 06)$.

\section{Expt 3}

Although pooled rumen contents were used to establish the cultures, two different fermentations were established on the hay 1 substrate. Initially, cultures 2 and 3 were characterized by high outputs of $\mathrm{Pr}$ and low outputs of Ac and cbl compared with those in cultures 1 and 4 (Fig. 2) and those in Expt 2. The introduction of barley as substrate increased the proportion of $\mathrm{Pr}$ and decreased that of Ac produced by each culture but there was no change in the culture differences for $5 \mathrm{~d}$. However, the molar proportion of $\operatorname{Pr}$ produced by culture 3 subsequently decreased to the level found in cultures 1 and 4 , followed $3 \mathrm{~d}$ later by an increase in cbl production. Day-to-day variations in cbl production on barley were large except for culture 2 .

Addition of Co was associated with small increases in cbl output (responses were smallest 

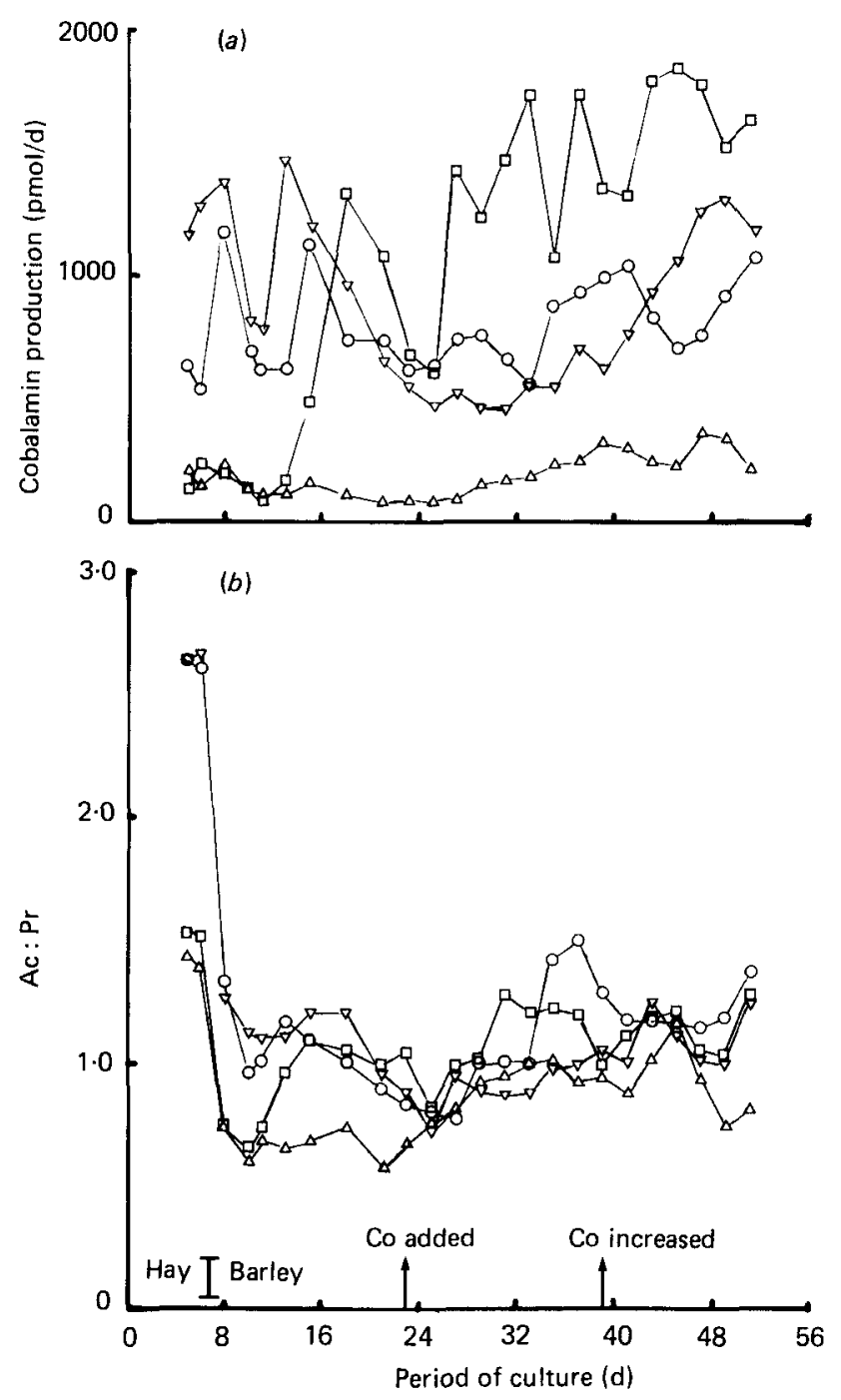

Fig. 2. Expt 3. Fermentation characteristics of cultures $1-4(O, \triangle, \square, \nabla$ respectively) given two cobalt-deficient substrates, hay (days 1-6) then barley (days 7-23). Cultures were supplemented with Co for two periods of $14 \mathrm{~d}$ each, at levels of 11.0 and $47.6 \mathrm{nmol} / \mathrm{d}$ (cultures 1 and 2) and 22.8 and $95.1 \mathrm{nmol} / \mathrm{d}$ (cultures 3 and 4). (a), Cobalamin production; $(b)$, acetate:propionate (Ac: $\mathrm{Pr}$ ).

in the culture (no. 2) with the lowest Ac:Pr) but much larger increases in analogue output in all cultures (Table 2). Co infusion was associated with increases in the proportion of Ac and hence Ac:Pr in the effluent VFA from the barley cultures (Fig. 2): the mean increase over the Ac proportion before supplementing the culture was 0.04 (SD 0.016) $(P<0.05$; $7 \mathrm{df}$ ). Over the entire period of barley feeding (Fig. 3) there was a linear relation between Ac proportion $(y)$ in the effluent and $\log (\mathrm{cbl})(x, \mathrm{pmol} / \mathrm{d})$ in which

$$
y=0.094+0.086 \text { (SD 0.0154) } x ; r 0.86 ; 10 \mathrm{df} \text {, }
$$

and between Ac proportion $(y)$ in the effluent and log analogue $(x, \mathrm{pmol} / \mathrm{d})$ in which

$$
y=0.045+0.089 \text { (SD 0.0194) } x ; r 0.80 ; 10 \mathrm{df} \text {. }
$$




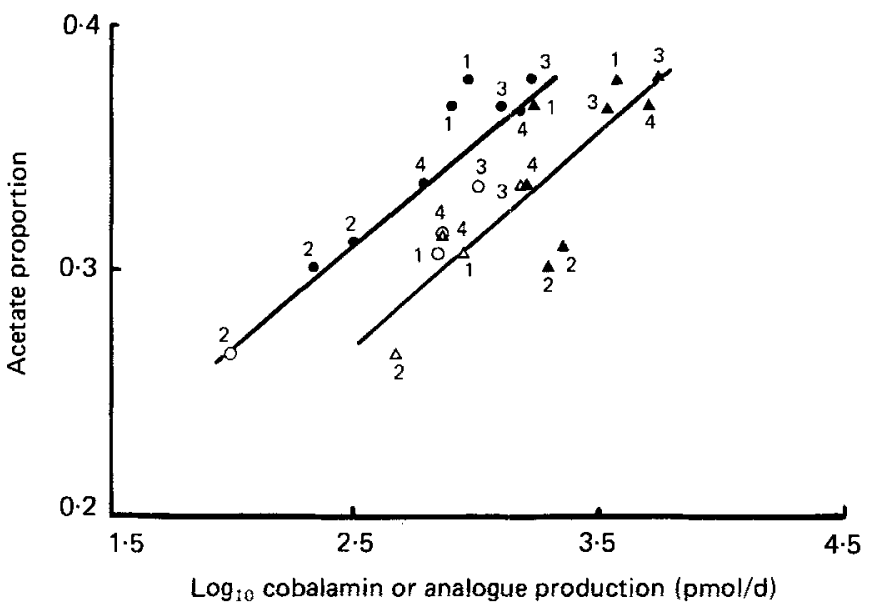

Fig. 3. Expt 3. The relation between $\log _{10}$ cobalamin $(O, O)$ or $\log _{10}$ analogue $(\Delta, \Delta)$ production and acetate as a proportion of total volatile fatty acids produced by four cultures (numbered 1,2, 3 and 4 ) given cobalt-deficient barley alone $(O, \triangle)$ and when supplemented with $C_{0}(\boldsymbol{O}, \Delta)$. Results are shown for each of the treatments detailed in Table 1 .

There was a marked reduction in Pr proportion following the addition of Co to culture 2 but no major changes in other VFA (Table 2).

\section{Expt 4}

Variation between cultures was a prominent feature of the fermentations established on the unsupplemented hay 2 (Table 2). Three of the four unsupplemented cultures produced low proportions of Ac and high proportions of $\mathrm{Pr}$, butyrate and valerate, giving $\mathrm{Ac}: \mathrm{Pr}$ which were quite uncharacteristic of a roughage fermentation (1.9 (SD 0.11)); the culture in vessel 1 was more characteristic $(\operatorname{Ac}: \operatorname{Pr} 2 \cdot 8)$. As in the other experiments, those unsupplemented cultures with low Ac:Pr produced less cbl: when supplemented with soils (providing $\mathrm{Co}$ ), their Ac: Pr values increased and analogue responses were large relative to cbl responses (Table 2).

When the results for all experiments were pooled, a positive linear relation between Ac proportion $(y)$ and $\log \mathrm{cbl}$ production $(x, \mathrm{pmol} / \mathrm{d})$ was found for the periods when the Co-deficient hay was used as substrate (Fig. 4):

$$
y=0 \cdot 131+0 \cdot 159(\text { SD } 0.0216) x ; r 0.88 ; 14 \mathrm{df} \text {. }
$$

\section{DISCUSSION}

The marked variations in fermentation characteristics between cultures given hay, evident in three of four experiments using different inoculation procedures, was totally unexpected. Previous workers using RUSITEC have reported normal VFA proportions and been sufficiently confident in the reproducibility of their cultures to use 'before and after' treatment comparisons within cultures without replication (Czerkawski \& Breckenridge, 1977, 1979; Wallace et al. 1980). Random variations and switches in the pattern of rumen fermentation have been recorded in individual sheep given a diet consisting predominantly of 'concentrates' (Hodgson \& Thomas, 1975). Czerkawski (1980) found day-to-day variations in RUSITEC cultures but there were no individual differences and the fluctuations coincided with those observed in vivo. An overall evaluation of our studies suggests a reason for culture individuality which may be of great physiological significance: we suggest 


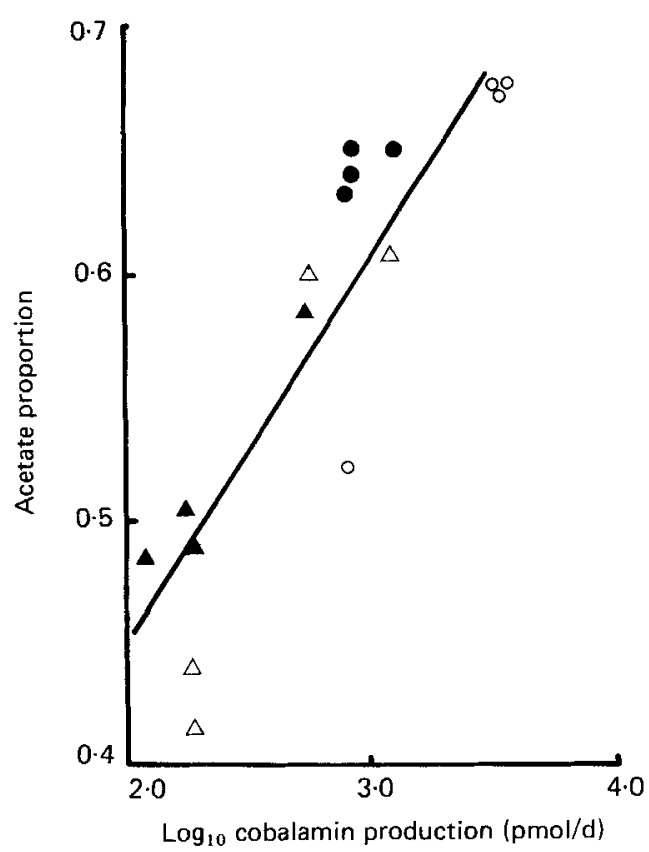

Fig. 4. Expts 1-4. The relation between $\log _{10}$ cobalamin production and acetate as a proportion of total VFA production from cultures given cobalt-deficient hay as a food substrate. Cobalamin production as estimated in Expts $1(O)$ and $2(0)$ was multiplied by a factor of $2 \cdot 29$ to correct for the diurnal fluctuations in cobalamin concentration in the culture vessel and hence outflow from the cultures to give values comparable to those obtained by effluent analysis in Expts $3(\Delta)$ and $4(\Delta)$ (McDonald, 1983).

that the fermentative activity of the cultured microbes was affected by the use of Co-deficient substrates.

The evidence of an effect of Co-deficiency on the cultured micro-organisms is as follows. First, there was a positive relation between $\mathrm{cbl}$ and Ac production and a negative relation with $\mathrm{Pr}$ in each experiment in which individuality was pronounced. Second, the provision of additional $\mathrm{Co}$ as cobalt nitrate to aberrant cultures was generally associated with a shift in fermentation from $\mathrm{Pr}$ to Ac when Co-deficient barley was fed (Table 2 and Fig. 2). Third, workers who have used substrates presumed to be adequate in Co have not encountered such individuality in their cultures. Support for an interaction between Co nutrition and the rumen microbial population is given by the early report of Gall et al. (1949) that certain microbial species declined in number when sheep were placed on a Co-deficient diet.

Although correlations between viable counts of bacteria and vitamin $\mathbf{B}_{12}$ concentrations have been reported in vivo (Dryden \& Hartman, 1971), it is unlikely that our correlation between cbl and Ac outputs arose from a common dependence on the size of the microbial mass. There was no evidence of low digestibility or total VFA production from cultures with low cbl and Ac outputs in Expts 3 and 4 (Table 2). Analogue outputs from the abnormal cultures were also low initially but no clear relations between analogue production and fermentation products were evident on unsupplemented hay (Expt 3), suggesting that it was the cbl status of the cultures which had the major influence on fermentation.

A possible explanation for the decrease in Ac: Pr in cultures with low vitamin $\mathbf{B}_{12}$ outputs is that Ac-producing species with high cbl requirements became less prevalent. Whereas most rumen micro-organisms produce Ac but not $\operatorname{Pr}$ as an end-product of fermentation, 
Selenomonas ruminantium, for example, produces both acids (Bryant, 1963). Three strains of $S$. ruminantium have been shown to contain analogues of vitamin $\mathrm{B}_{12}$ but no $\mathrm{cbl}$ in pure cultures (Dryden \& Hartman, 1971). Thus species such as $S$. ruminantium might prosper in cultures given Co-deficient substrates, with a concomitant increase in $\operatorname{Pr}$ production. Changes in the protozoal population may also be involved since they are believed to contain little cbl (Porter, 1961) and are associated with changes in $\operatorname{Pr}$ production both in vivo (Eadie et al. 1970) and in vitro (Wallace et al. 1980).

The next question is how could such individuality arise in an apparently uniform set of four in vitro cultures? There is no reason to suppose that there were large sustained differences in the known exogenous or endogenous supply of Co to the cultures. We suggest, therefore, that small differences in the initial supplies of Co from the inoculum, culture vessel or tubes supplying artificial saliva, determined the species which became established on Co-deficient substrates. The very act of displacing half the microbial population from the feed matrix each day may have enhanced its vulnerability. The fact that Co supplementation did not quickly increase cbl or Ac outputs in any aberrant culture also supports the suggestion that the final between-culture differences reflected the composition of the microbial population rather than its Co supply. The large responses in analogue but not cbl production when Co was infused (Expts 3 and 4 in Table 2) are consistent with the possibility that the cbl-containing species had been depleted in number.

The use of Co-depletion and repletion of RUSITEC cultures to assess the availability of a Co source and factors affecting partition of available Co between $\mathrm{cbl}$ and analogues is clearly not to be recommended, because of the likelihood of unstable fermentations. It is possible that the technique could be used under conditions of generous Co supply. Nevertheless, the occurrence of such changes in fermentation raises interesting possibilities. If Co-deficient diets cause shifts in rumen populations and enhance Pr synthesis in vivo, as they appear to do in vitro, this could initially improve feed conversion efficiency. Eventually, however, the functional vitamin $\mathbf{B}_{12}$ deficiency in the host tissues, believed to involve an impaired ability to metabolize Pr due to a co-enzyme (adenosyl cbl) deficiency (Marston et al. 1961), might be exacerbated. Marston et al. (1972) concluded from studies of VFA concentrations in the rumen of two sheep on a Co-deficient hay, with and without Co supplements, that the proportions of Ac and Pr present were not influenced by Co supply. However, it is noteworthy that both animals began the experiment with low $\mathrm{Ac}: \mathrm{Pr}$ $(2: 1)$. Further studies of rumen fermentation on Co-deficient diets are clearly necessary.

The authors are indebted to Dr David Purves and his colleagues of the East of Scotland College of Agriculture, Edinburgh for cobalt analyses, and to Mrs E. Herbert for her skilled technical support.

\section{REFERENCES}

Babior, B. M. (editor) (1975). In Cobalamin Biochemistry and Pathophysiology, pp. 141-212. London:John Wiley. Bryant, M. P. (1963). Journal of Animal Science 22, 801-813.

Czerkawski, J. W. (1980). Proceedings of the Nutrition Society 39, 72 A.

Czerkawski, J. W. \& Breckenridge, G. (1977). British Journal of Nutrition 38, 371-384.

Czerkawski, J. W. \& Breckenridge, G. (1979). British Journal of Nutrition 42, 217-228.

Dryden, L. P. \& Hartman, A. M. (1971). Journal of Dairy Science 54, 235-246.

Dryden, L. P., Hartman, A. M., Bryant, M. P., Robinson, I. M. \& Moore, L. A. (1962). Nature 195, $201-202$.

Eadie, J. M., Hyldgaard-Jensen, J., Mann, S. O., Reid, R. S. \& Whitelaw, F. G. (1970). British Journal of Nutrition 24, 157-177.

Gall, L. S., Smith, S. E., Becker, D. E., Stark, C. N. \& Loosli, J. K. (1949). Science 109, 468 469.

Gawthorne, J. M. (1970). Australian Journal of Experimental Biology and Medical Science 48, 285-292.

Green, R. (1980). Methods in Enzymology 67, 99-108.

Hodgson, J. C. \& Thomas, P. C. (1975). British Journal of Nutrition 33, 447-456.

McDonald, P. G. (1983). The biological availability of cobalt to ruminants. PhD Thesis, University of Edinburgh. 
McDonald, P. G. \& Suttle, N. F. (1983). Occasional Publication of The British Society of Animal Production no. 7, pp. 147-148.

McDougall, E.I. (1948). Biochemical Journal 43, 99-109.

Marston, H. R., Allen, S. H. \& Smith, R. M. (1961). Nature 190, 1085-1091.

Marston, H. R., Allen, S. H. \& Smith, R. M. (1972). British Journal of Nutrition 27, 147-151.

Porter, J. W. (1961). In Digestive Physiology and Nutrition of the Ruminant, p. 226 [D. Lewis, editor]. London: Butterworths.

Poston, J. M. \& Stadtman, T. C. (1975). In Cobalamin Biochemistry and Pathophysiology, pp. 111-140 [B. M. Babior, editor]. London: John Wiley.

Sutton, A. L. \& Elliot, J. M. (1972). Journal of Nutrition 102, 1341-1346.

Wainman, F. W., Blaxter, K. L. \& Pullar, J. D. (1970). Journal of Agricultural Science, Cambridge 74, 311-314.

Wallace, R. J., Cheng, K. J. \& Czerkawski, J. W. (1980). Applied and Environmental Microbiology 40, $672-674$. 